\title{
„Fehlende Regelung im Urheberrecht höhlt Kultur- und Bildungsinfrastruktur der Öffentlichen Bibliotheken aus!“
}

\section{Über 600 Bibliotheksleitungen fordern in einem Offenen Brief den ungehinderten Zugang zu E-Books}

https://doi.org/10.1515/bd-2021-0027

Immer mehr Bücher werden heute als E-Book digital veröffentlicht und gelesen. Der digitale Zugang ist gerade in Zeiten von Corona und geschlossenen Bibliotheksgebäuden oft die einzige Möglichkeit für Bürger^innen, an Bücher, Informationen und Medien heranzukommen. Doch beim sogenannten „E-Lending“ - also der temporären Bereitstellung einer Nutzungslizenz für ein elektronisches Buch wird den Bibliotheken ein Riegel vorgeschoben. 70 Prozent der E-Book-Titel der Spiegel-Bestsellerliste (Belletristik und Sachbücher) werden Bibliotheken bis zu einem Jahr lang vorenthalten.

In einem Offenen Brief, den bislang bereits über 600 Bibliotheksleitungen unterschrieben haben, fordert der Deutsche Bibliotheksverband e.V. (dbv) die Abgeordneten des Deutschen Bundestages nun auf, sich dafür einzusetzen, dass Bibliotheken ihrem Kultur- und Bildungsauftrag durch den umfassenden Verleih auch von elektronischen Büchern nachkommen können.

Den Offenen Brief können Sie hier abrufen: https://www.bibliotheksverband. de/dbv/kampagnen-und-aktionstage/e-medien-in-der-bibliothek/offener-brief.

Dazu Prof. Dr. Andreas Degkwitz, Bundesvorsitzender des dbv: „Der Deutsche Bibliotheksverband vertritt bundesweit 11 Mio. Bibliotheksnutzer`innen. Sein zentrales Anliegen ist es, Bürger`innen ungehinderten Zugang zu Informationen, Bildung und Medien zu ermöglichen. Denn der freie Zugang zu Wissen und Information unabhängig von Bezahlschranken ist ein Grundrecht. Doch das seit Jahren fehlende Verleihrecht für E-Books im Urheberrecht höhlt die Kultur- und Bildungsinfrastruktur der Öffentlichen Bibliotheken aus. Anlässlich des aktuell vorliegenden Gesetzentwurfes zur Änderung des Urheberrechtsgesetzes ist es daher unerlässlich, endlich eine entsprechende gesetzliche Regelung aufzunehmen. Der dbv und die über 600 Unterzeichner`innen des Offenen 
Briefes fordern daher die Politik auf, hier endlich die notwendige Grundlage zu schaffen."

Die Kernforderungen der Bibliotheken lauten:

1. E-Books sollen dem gedruckten Buch in allen Bereichen rechtlich gleichgestellt werden - wie dies bereits bei der reduzierten Mehrwertsteuer und der Buchpreisbindung erzielt wurde - so auch beim E-Lending. Dazu ist eine gesetzlich geregelte Grundlage für faire Lizenzmodelle durch eine entsprechende Aktualisierung des Urheberrechtsgesetzes nötig.

2. Die Bibliothekstantieme soll auf elektronische Werke ausgeweitet werden, damit Autor`innen auch für das E-Lending wie bei gedruckten Büchern angemessen vergütet werden. Im Gegenzug dazu müssen den Bibliotheken die gleichen Nutzungsrechte für elektronische wie für gedruckte Werke eingeräumt werden.

Mehr Informationen zu der Kampagne \#BuchistBuch und zum E-Lending finden Sie hier: https://www.bibliotheksverband.de/dbv/kampagnen-und-aktionstage/ e-medien-in-der-bibliothek.html.

\section{Zum Hintergrund}

Verlage verhandeln für den Verleih ihrer E-Books mit - für Bibliotheken tätigen Firmen wie divibib GmbH („Onleihe“) oder Overdrive Inc. („Libby“) spezielle Bibliothekslizenzen zu unterschiedlichen Konditionen. Diese Firmen stellen auch die technischen Plattformen und den Support bereit. Bibliotheken schließen mit diesen Firmen Verträge über die von ihnen angebotenen Lizenzen ab. Sie wählen nach rein fachlichen Kriterien, welche Titel zu ihrem Bestand passen und für ihre jeweiligen Zielgruppen sinnvoll erscheinen. Das Verfahren ist das gleiche wie beim gedruckten Buch: Es gibt nur eine Ausleihe pro Person, feste Leihfristen und die E-Books, die gerade verliehen sind, können erst dann vom / von der nächsten Leser*in genutzt werden, wenn sie „zurückgegeben“ wurden. Bibliotheken bieten also keine kommerzielle „Flatrate“, bei der man für eine bestimmte Summe unbegrenzt auf E-Books zugreifen könnte. Die Ausleihe von E-Books ermöglicht Bürger`innen, die sich den Kauf von Büchern nicht leisten können, den Zugang zu aktueller Literatur. Der Buchhandel wird dabei ebenso wenig geschädigt wie bei der Ausleihe gedruckter Bücher.

\section{Der Deutsche Bibliotheksverband e.v. (dbv)}

Der Deutsche Bibliotheksverband e.V. (dbv) vertritt mit seinen mehr als 2.100 Mitgliedern bundesweit über 9.000 Bibliotheken mit 25.000 Beschäftigten und 11 Mio. Nutzer^innen. Sein zentrales Anliegen ist es, Bibliotheken zu stärken, damit sie allen Bürger^ freien Zugang zu Informationen ermöglichen. Der Verband setzt 
sich ein für die Entwicklung innovativer Bibliotheksleistungen für Wissenschaft und Gesellschaft. Als politische Interessensvertretung unterstützt der dbv die Bibliotheken insbesondere auf den Feldern Informationskompetenz und Medienbildung, Leseförderung und bei der Ermöglichung kultureller und gesellschaftlicher Teilhabe für alle Bürger*innen.

Mehr Informationen finden Sie unter www.bibliotheksverband.de.

\section{Pressekontakt}

\section{Kristin Bäßler}

Deutscher Bibliotheksverband

Leitung Kommunikation / Pressesprecherin

Tel.: + 49 (0)30 6449899 25 | E-Mail: baessler@bibliotheksverband.de 